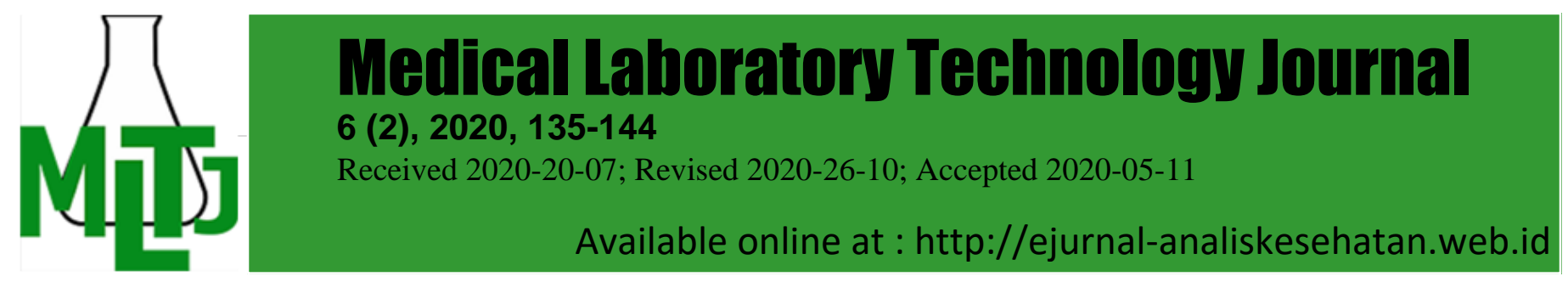

\title{
Role of CD44 and CD8 in Colorectal Adenocarcinoma Metastatic
}

\author{
Baiq Ratna Kumaladewi, *Willy Sandhika, Heryawati
}

\author{
Department of Anatomic Pathology, Faculty of Medicine, Universitas Airlangga/ \\ Dr. Soetomo Hospital, Surabaya, Indonesia. \\ *Email: willysand@fk.unair.ac.id \\ DOI: $10.31964 / \mathrm{mltj} . v 6 \mathrm{i} 2.314$
}

\begin{abstract}
Colorectal adenocarcinoma is one of the most prevalent malignancies worldwide. The presence of regional lymph node metastases is an important prognostic indicator in colorectal adenocarcinoma. CD44 is a transmembrane protein and a cancer stem cell biomarker that plays a role in tumor proliferation, metastasis, and recurrence. The tumor microenvironment has a vital role in the stage of tumor development. CD8 is one of microenvironment's component which kills the tumor cells. This study aimed to analyze the expression of CD44 and CD8 and investigated their association with colorectal adenocarcinoma metastatic. This study's benefit is that the resulting study can be used as a prognostic factor in colorectal adenocarcinoma. This study included 56 paraffin blocks of patients diagnosed as colorectal adenocarcinoma during January 2017-December 2018 in Anatomic Pathology Laboratory Dr. Soetomo Hospital Surabaya. The paraffin blocks were sliced and stained with immunohistochemistry for CD44 and CD8. The parameters were a positive percentage of tumor cell and lymphocyte immunoreactivity against CD44 and CD8 antibodies. The percentage of CD44 is higher in the metastatic group, and the percentage of CD8 is higher in the non-metastatic group. There was a significant negative correlation between CD44 and CD8 expression in regional lymph nodes metastatic. These results indicated that CD44 and CD8 have a vital role in the incidence of metastasis.
\end{abstract}

Keywords: CD44; CD8; lymph node metastatic

\section{INTRODUCTION}

Colorectal carcinoma is the third most common malignancy globally, with an increased incidence in the elderly. Every year, colorectal carcinoma is diagnosed by more than 940,000 people, and around 500,000 people die from this cancer (Holah et al., 2017). It estimated that there are 1.23 million new cases globally, which is about $9.7 \%$ of all new cases of malignancy. This malignancy is placed third in males after lung, prostate, and gastric malignancies (Hamilton et al., 2010; Rawla et al., 2019).

The data gathered in the Anatomy Pathology laboratory Dr. Soetomo Surabaya show an increase in the number of new diagnoses of colorectal carcinoma cases each year, namely 104 cases in 2012, 143 cases 2013, and then 169 cases in 2014.

The role of cancer stem cells within tumor development began to be widely studied; CD44 is a marker of stem cells in colorectal adenocarcinoma. CD44 is a transmembrane protein which is also part of cell adhesion molecules that plays a role in cell-to-cell communication and interactions with the extracellular matrix and has a role in tumor development, proliferation, and metastasis in which affect the prognosis and survival (Chen et al., 2018; Holah et al., 2017).

Cancer stem cells are characterized by their resistance to anti-cancer therapy and trigger tumor metastases and recurrence. Radiation and chemotherapy can Corresponding Author: Willy Sandhika Department of Anatomic Pathology, Faculty of Medicine, Universitas Airlangga/Dr. Soetomo General Hospital, Surabaya, Jl. Mayjen Prof. Dr. Moestopo No 47, Surabaya, East Java, 60132, Indonesia. Email: willysand@fk.unair.ac.id 
currently kill most cancer cells but often do not eliminate cancer stem cells protected by specific resistance mechanisms (Magee et al., 2012).

CD44 is a transmembrane glycoprotein. The expression of CD44 is also regulated in the sub-population of cancer cells. It is recognized as a molecular marker for cancer stem cells, one of which is in colon cancer (Chen et al., 2018). Previous studies have shown that CD44 expression can be influenced by the degree of differentiation. A low degree of differentiation indicates that a positive CD44 percentage will be higher. Likewise, with its expression related to lymph node metastasis, high CD44 expression has a worse prognosis (Ma et al., 2019; Zhao et al., 2015).

The tumor cells' ability to express CD44 to metastasize is influenced by the micro-environment around the tumor, including stromal cells, extracellular matrix, blood vessels, and immune cells. Immune cells are one part of the micro-environment. One of the immune cells that play a role in tumor development is CD8, which functions as a cytotoxic T lymphocyte (CTL) that will kill tumor cells. The low density of CD8 will affect the risk of recurrence, metastasis, and survival in colorectal adenocarcinoma (de Vries et al., 2016).In previous research shows that there is a correlation between high frequency of CD8 positive lymphocyte infiltration and lack of lymph node involvement in rectal cancer (Daster et al., 2014).

Previous research involving these two markers has not been conducted. Thus, this research is vital to comprehend the role of both markers in the metastatic process in colorectal adenocarcinoma.

\section{MATERIALS AND METHOD}

This study had approved by the Health Research Ethics Committee of Dr. Soetomo General Hospital, Surabaya (Ethical Clearance no. 1561/KEPK/X/2019). This study was an analytic observational study with a cross-sectional approach, using paraffin-embedded from the operating tissue of patients with colorectal adenocarcinoma, which were established based on histopathological features in the Anatomical Pathology Installation of Dr. Soetomo Surabaya period January 2017 December 2018. The total sample of 56 samples consisted of 2 groups, namely colorectal adenocarcinoma with regional lymph node metastases and colorectal adenocarcinoma without regional lymph node metastases. Thus, the total sample of each group was 28 paraffin-embedded.

This tissue was cut into $4 \mu \mathrm{m}$ sections, deparaffinized three times with xylol for 5 minutes each, and rehydrated through graded alcohol. Antigen retrieval was achieved by microwave treatment in sodium citrate buffer $(\mathrm{pH} \mathrm{6,0)}$ for 10 minutes. These tissue sections were then incubated with monoclonal antibodies for HCAM (DF1485: sc-53534; dilution 1:250; Santa Cruz Biotechnology) and CD8 (4B11; dilution 1:50; Leica Biosystems) followed by the secondary antibody for 10 minutes at room temperature. The section was then counterstained with hematoxylin and dehydrated with alcohol.

The study used CD44 and CD8 immunohistochemistry in adenocarcinoma colorectal paraffin blocks with and without regional lymph node metastases. CD44 was examined with which was positively stained on the membrane and cytoplasm of tumor cells. CD8 was examined with which was positively stained on the lymphocyte cell membrane.

CD44 expression was assessed by calculating the mean percentage in the five densest fields of view by dividing the number of tumor cells stained brown on the membrane and cytoplasm by the number of tumor cells in the field of view. 
$\%$ CD44 (one field of view) $=\underline{\text { number of tumor cells stained brown }} \times 100 \%$

Total number of tumor cells

Assessment of CD8 expression in each sample group was done by calculating the mean percentage in the five densest fields of view by dividing the number of lymphocyte cells stained brown on the membrane by the number of lymphocyte cells in that field of view.

$\%$ CD8 (one field of view) = number of lymphocyte cells stained brown X 100\%

Total number of lymphocyte cells

\section{RESULTS AND DISCUSSION}

Data analysis used to determine differences in CD44 and CD8 expression with regional lymph node metastasis status in colorectal adenocarcinoma is an independent T-test. To find out the correlation between CD44 and CD8, the study uses the Pearson correlation test. The statistical test was significant if $p<0.05$.

CD44 expression in this study is reflected in the membrane and cytoplasm of tumor cells (Figure 1). The data obtained from this study showed that the mean CD44 expression in the metastatic group was 61.8 \pm 26.7 , while in the non-metastatic group, it was $41.8 \pm 27.4$. The differences between the two groups were then analyzed using an independent T-test. The statistical analysis results showed a significant difference between CD44 expression in colorectal adenocarcinoma with metastases and without regional lymph node metastases with $p=0,008(p<0.05)$. These data showed a higher expression of CD44 in colorectal adenocarcinoma with metastases than without regional lymph node metastases (Table 1).

The correlation between CD44 expression and colorectal adenocarcinoma metastatic status statistically tested using the Spearman correlation test. The analysis showed a significant correlation where the Spearman correlation coefficient was 0.348 with $p=0.003(p<0.05)$. These results indicate that the higher the expression of CD44 is in line with the increase in regional lymph node metastasis status (Table 2).

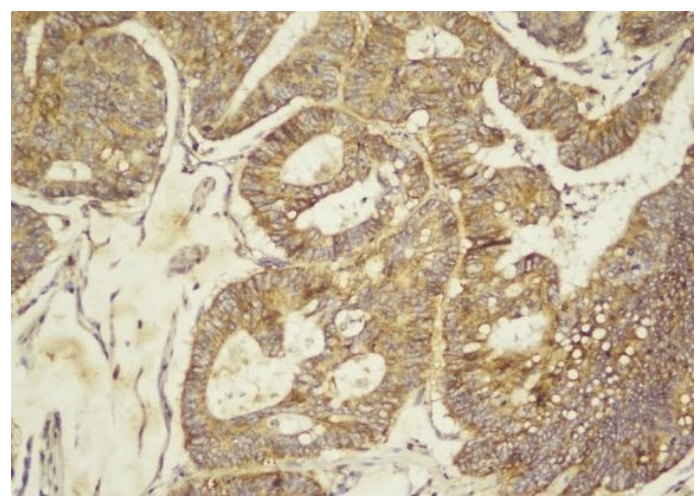

Figure 1. Expression of CD44 on Colorectal Adenocarcinoma Tumor Cells.CD44 Streaked Brown on the Membrane and Cytoplasm of Tumor Cells Magnification 200x

Table 1. CD44 Expression in Colorectal Adenocarcinomas

\begin{tabular}{lccccc}
\hline Metastatic status & Mean & SD & Min & Maks & $\mathrm{p}$ \\
\hline Metastatic & 61,8 & 26,7 & 6,57 & 91,72 & 0,008 \\
Non-metastatic & 41,8 & 27,4 & 2 & 90,69 & \\
\hline
\end{tabular}


Tabel 2. Association between CD44 Expression with Metastatic Status

\begin{tabular}{ccc}
\hline & & CD44 expression \\
\hline Metastatic status & $\mathrm{rs}_{\mathrm{s}}$ & 0,348 \\
& $p$ & 0,003 \\
& $\mathrm{n}$ & 56 \\
\hline
\end{tabular}

CD8 expression in this study was reflected in the lymphocyte cell membrane (Figure 2). This study showed that the mean CD8 expression in the metastatic group was $30.8 \pm 13.1$, while the non-metastatic group was $45.3 \pm 11.9$. The differences between the two groups were then analyzed using an independent T-test. The statistical analysis results showed a significant difference between CD8 expression in colorectal adenocarcinoma with metastases and without regional lymph node metastases with $\mathrm{p}=0,01 \quad(\mathrm{p}<0.05)$. These data showed that CD8 expression in colorectal adenocarcinoma with metastases is lower than its expression in the area without regional lymph node metastases (Table 3).

The correlation between CD8 expression and metastatic status in colorectal adenocarcinoma statistically tested using the Spearman correlation test. The analysis showed a significant correlation where the Spearman correlation coefficient was 0.544 with $p=0.001 \quad(p<0.05)$. These results indicate that the higher the expression of CD8 is in line with the decrease in the metastatic status of regional colorectal adenocarcinoma lymph nodes (Table 4).

The Pearson correlation test results showed a significant relationship between CD8 and the metastatic status of regional lymph nodes $(p=0.001)$ with a correlation coefficient of -0.508 . These results indicate that the higher CD8 expression is in line with the decreased status of regional lymph node metastases.

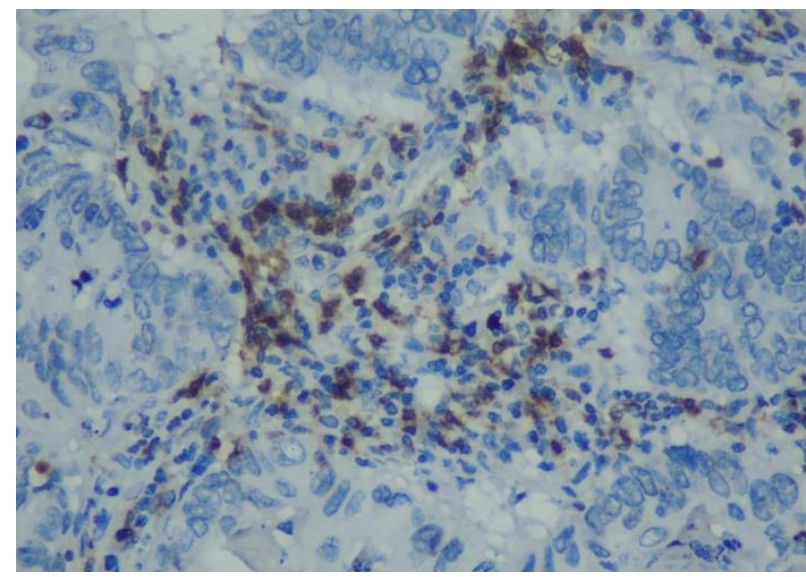

Figure 2. CD8 Expression in Colorectal Adenocarcinoma. CD8 was Streaked Brown in $70 \%$ of the Lymphocyte Cell Membrane within Tumor Cells. 400x Magnification

Table 3. CD8 Expression in Colorectal Adenocarcinomas

\begin{tabular}{cccccc}
\hline Metastatic status & Mean & SD & Min & Maks & $\mathrm{p}$ \\
\hline Metastatic & 30,8 & 13,1 & 4,77 & 60,37 & 0,001 \\
Non-metastatic & 45,3 & 11,9 & 4,1 & 60,94 & \\
\hline
\end{tabular}


Tabel 4. Association between CD8 Expression with Metastatic Status

\begin{tabular}{lcc}
\hline & & CD8 expression \\
\hline Metastatic status & $r_{s}$ & $-0,544$ \\
& $p$ & 0,001 \\
& $\mathrm{n}$ & 56 \\
\hline
\end{tabular}

The correlation between CD44 and CD8 expression in colorectal adenocarcinoma with and without regional lymph node metastases statistically tested using the Pearson correlation test. The analysis showed that there was a significant correlation where the Pearson correlation coefficient was -0.316 with a $p$-value $=0.018$ $(p<0.05)$, which means that the higher the CD44 expression, the lower the CD8 expression (Table 5).

Tabel 5. Correlation CD44 and CD8 Expressions

\begin{tabular}{ccc}
\hline & & CD8 expression \\
\hline CD44 expression & $r$ & $-0,316$ \\
& $p$ & 0,018 \\
& $r$ & 56 \\
\hline
\end{tabular}

This study's results are consistent with several previous studies, which stated a significant correlation between CD44 expression and lymph node metastasis status in colonic adenocarcinoma, a higher CD44 expression obtained in tumor cells with lymph node metastasis (Holah et al., 2016). Research by Zhao et al. also showed a correlation of CD44 expression with lymph node metastasis and TNM stage in colorectal carcinoma, where the higher stage $\mathrm{N}$ showed higher CD44 expression in primary tumors (Zhao et al., 2015).

Another study conducted by $\mathrm{Li}$ et al. looked at the expression of CD44 with CD44 variant exon 6 , where there was a significant relationship between both expression and lymph node metastasis, degree of differentiation, stage of Dukes, and survival rate of 5 years. This study shows that colorectal carcinoma patients with high CD44 have a 5-year life expectancy of 52.78\%, while those with negative CD44 show a better life expectancy of $80.9 \%$ (Li et al., 2013; Ma et al., 2019). Another study on breast carcinoma by Ma and Jiang showed a higher CD44 expression in breast carcinomas with lymph node metastases than without metastases (Ma and Jiang, 2013).

Several previous studies have shown different results that high CD44 expression is not associated with the incidence of lymph node metastasis and shows better expression in colorectal carcinoma (Du et al., 2008). Research by Chen et al. showed no significant relationship between CD44 expression and grade, stage, and prognosis (Chen et al., 2011). The difference in this study results could be due to differences in the isoform variant of CD44 and also influenced by its binding to the extracellular matrix components ( $\mathrm{Ma}$ et al. 2019). Other factors can be due to differences in the type of antibody, the number of samples, the staining process, and immunohistochemical reporting (Chaitra et al., 2018).

The role of CD44 in cancer progression is influenced by the microenvironment around the tumor, including immune cells, fibroblasts, vascular endothelial cells, extracellular matrix components, and various cytokines and chemokines. Various 
cytokines that affect the action of CD44 include IL-8, G-CSF which play a role in tumor proliferation, angiogenesis, and metastasis, and TGF- $\beta$, which is involved in the EMT mechanism associated with tumor cell invasion and metastasis. One of the chemokine molecules that affect the action of CD44 is CXCL12. The interaction between CXCL12 and CXCR4 will affect the role of CD44 in metastasis of colorectal carcinoma. Inhibition of the CXCL12-CXCR4 interaction will reduce the expression of CD44 in tumor cells (Chen et al., 2018; Ma et al., 2019). CD44 through CXCR4 can also affect the immune system around tumor cells. CXCR4 protein will attract dendritic cells to come into the environment around the tumor. Dendritic cells can suppress anti-tumor immunity through cytotoxic T-lymphocytes inactivation(Xu et al., 2015).

CD44 is a receptor located in the cell membrane, which then binds with hyaluronan acid (HA) as a ligand located on the extracellular matrix (Chen et al., 2018). The interaction between CD44 and HA modifies the extracellular matrix component (ECM) to trigger tumor cell invasion by stimulating the production of MMP2 and MMP9. Furthermore, MMP2 can degrade type IV collagen as the main component that forms the structure of cell membranes. If the barrier can be passed, the tumor cells will develop metastasis to lymph nodes and distant organs. MMP9 can also trigger metastasis by triggering TGF $\beta$ activity through interactions with CD44. TGF $\beta$ / SMAD signals induce EMT so that metastases can occur (Ma et al., 2019).

The role of CD44 in cancer progression is influenced by the micro-environment surrounding the tumor, including immune cells, fibroblasts, vascular endothelial cells, extracellular matrix components, and various cytokines and chemokines [29]. Various cytokines that affect the work of CD44 include IL-8, G-CSF, which role is affecting tumor proliferation, angiogenesis, and metastasis, and also TGF- $\beta$, which participate in the EMT mechanism associated with invasion and metastasis of tumor cells. One chemokine molecule that affects CD44's action is CXCL12 (Ma et al., 2019).

Cytotoxic T Lymphocytes (CTL) are lymphocytes that can induce damage and kill infected cells or tumor cells (Abbas et al., 2015). CD8 (Cluster of Differentiation 8) is a glycoprotein that functions as a co-receptor for T Cell Receptors, mainly expressed on cytotoxic T-cells' surface. Some studies state that the infiltration of CD8 ${ }^{+} \mathrm{T}$ cells in tumors and their environment will provide a better prognosis (Barnes \& Amir, 2017; West et al., 2015; Ziai et al., 2018). The infiltration of $\mathrm{CD}^{+}{ }^{+}$T cells in tumors itself happens at the intertumoral location (between tumor cells) or stromal (between tissues and blood vessels around the tumor) (Ziai et al., 2018).

Immune cells are part of the tumor microenvironment, which influences the development of tumor cells. CD8 ${ }^{+}$T-cells are a component of immune cells that cause tumor cells' death through an apoptotic mechanism by releasing granzymes and perforin (Barnes and Amir., 2017; Jackute et al., 2015).

A study by ldos et al. demonstrated an association between CD8+ T lymphocytes' presence and the survival of colorectal cancer patients. The high number and density of CD8+ T-lymphocytes have better survival (Idos et al., 2020).

Research conducted by West et al. examined that the presence of $\mathrm{CD}^{+}$is associated with improved patient life expectancy compared to low infiltration from $\mathrm{CD}^{+}$. This condition depends on the number of cytotoxic $\mathrm{T}$ cells in primary tumors associated with reduced likelihood of metastasis and increasing life expectancy of colorectal cancer patients (Iseki et al., 2018; West et al., 2015).

A study conducted by Kedr et al. stated that a significant decrease in CD8 ${ }^{+} \mathrm{T}$ cell was associated with a poor prognosis with increased tumor development and metastatic events (Khedr et al., 2016). 
The literature reviews show that differences in mutation loads cause differences in CD8 expression in colorectal carcinoma. These high mutation loads can produce high antigenicity by regulating antigens recognized by the immune system. Colorectal carcinomas with MSI phenotype show increased immunogenicity compared to colorectal carcinomas with MSS mutations. This is indicated by the presence of more dominant $\mathrm{CD}^{+}$cytotoxic $\mathrm{T}$ cells found in colorectal carcinomas with higher immunogenicity (de Vries et al., 2016).

This study found a significant negative correlation between CD44 expression and CD8 expression in colorectal adenocarcinoma. Considering some studies, CD44 expression positively correlates with FOXP3, where FOXP3 is one of the T regulator markers. $T$ regulator is an immunosuppressive whose function is to regulate the immune reaction's balance and maintain tolerance of the antigen itself. In certain circumstances, $T$ reg will suppress the ability of CD8 cytotoxic T cells against tumor cells. This situation involves TGF $\beta$ and IL10 (Chen et al., 2005; Peng et al., 2019).

The immune response is a very complex system, and it is difficult to explain how many immune factors greatly influence the presence of stem cells and cancer cells. The initial phase is the elimination phase, where the innate and adaptive immune systems work to destroy cancer cells. This requires a balance of anti-tumor immune responses such as CD8 T cells, CD4 T cells, and NK cells. In the equilibrium phase, there is a balance between anti-tumor and pro-tumor factors with CD8 selection, where $T$ reg plays a role in inhibiting APC, CD8, NK cells, and CD4 cells. This process will inhibit recognition and destruction by immune cells so that carcinoma cells will be released and develop in the final phase. In a continuous and prolonged activity, CD8 T cells will experience fatigue, then CTLA-4 and PD1 on CD8 will provide inhibitory signals that cause a decrease in CD8 (Dushyanthen et al., 2015).

The body's immune response can have anti-tumor and pro-tumor roles. Tumor cells, in this case, cancer stem cells, will activate macrophages by releasing IL-10. Macrophages have a role in adaptive immunity by producing cytokines, namely IL-1, which triggers Th1 to produce IFNy (Abbas et al., 2015). Effective anti-tumor immunity is thought to be driven mainly by interferon- $\mathrm{Y}$ (IFNY). Dendritic cells express costimulatory molecules and inflammatory cytokines such as IL-12, IL-23, and IL-1 that trigger the response of CD4 ${ }^{+} \mathrm{T}$ cells secreting IFNy and cytotoxic T lymphocytes (Wang et al. 2017).

$\mathrm{CD}^{+}$T-cells can recognize and bind to $\mathrm{MHC}$ II on APC, while CD8 ${ }^{+}$with $\mathrm{MHC}$ I, which plays a role in tumor cells' death through apoptosis by releasing granzyme and perforin. NK cells, with the help of APC and $\mathrm{CD4}^{+}$, are also able to recognize and eliminate tumor cells. CTL's role in killing tumor cells is often not able to eliminate the cancer stem cells so that they can continue to develop and the occurrence of metastases (Magee et al., 2012). NK cells recognize molecules expressed by tumor cells and will be activated if the target cells do not have MHC class I molecules. Therefore NK cells have a mechanism to kill - negative MHC class I (class I MHCnegative tumors) (Abbas et al., 2015).

TAM influences the process of migration and metastasis through the extracellular matrix. TAM produces proteases and Matrix Metalloproteinase (MMP), which cause degradation of ECM and basement membrane. Besides, the presence of epithelial-mesenchymal transition (EMT) has a role in metastasis, resulting from the interaction of tumor cells with TAM. TAM also plays a role in forming a premetastatic niche (Yang et al., 2017).

Colorectal carcinoma with the MSI phenotype showed more CD8 ${ }^{+}$cytotoxic T lymphocyte (CTL) infiltration. CTL induces cancer cell death by relying on the bonds 
between the Fas-Fas ligands. CTL expresses ligands so that Fas on the surface of tumor cells will bind to ligands on CTL to undergo apoptosis. The CD44 molecule functions to bind Fas to prevent the interaction between Fas and Fas ligands (Ma et al., 2019).

This study indicates that an increase in CD44 expression in tumor cells and a decrease in CD8 expression in lymphocytes around the tumor are associated with an increased incidence of metastases in colorectal adenocarcinoma. This research is expected to be useful as a prognostic, predictive factor, and progression of colorectal cancer and subsequent immunotherapy development. This study's drawback is that the data used are limited to tumor cells with regional lymph node metastases only, not examining distant organ metastases.

\section{CONCLUSION}

The expression of CD44 and CD8 is an important marker for determining the incidence of metastasis in colorectal carcinoma to be used as a prognostic factor for cancer development. This research can be used as a reference for future research regarding the role of cancer stem cells and microenvironment components.

\section{ACKNOWLEDGMENT}

The authors thanks Budi Utomo, MD from Department of Community Medicine for sharing statistical aspect of this study. The authors also acknowledge all of lectures in Department of Anatomical Pathology, Faculty of Medicine, Universitas Airlangga for the guidance in finishing this study.

\section{CONFLICT OF INTEREST}

The authors have no conflict of interest to disclose.

\section{REFERENCE}

Abbas, A. K. Lichtman, A. H. Pillai, S. (2015) Basic immunology, functions and disorders of the immune system (5th ed), Elsevier. pp. 218-223.

Barnes, T.A., \& Amir, E. (2017). HYPE or HOPE: the prognostic value of infiltrating immune cells in cancer. British Journal of Cancer, 117(4), 451-460. https://doi.org/10.1038/bjc.2017.220

Chaitra, L. P., Prashant, A., Gowthami, C. S., Hajira, B., Suma, M. N., Mahesh, S. S., Manjunath, G. V., \& Sheeladevi, C. S. (2019). Detection of cancer stem cellrelated markers in different stages of colorectal carcinoma patients of Indian origin by immunohistochemistry. Journal of cancer research and therapeutics, 15(1), 75-81. https://doi.org/10.4103/jcrt.JCRT_991_16

Charan, J., \& Biswas, T. (2013). How to calculate sample size for different study designs in medical research?. Indian Journal of Psychological Medicine, 35(2), 121-126. https://doi.org/10.4103/0253-7176.116232

Chen, C. Zhao, S. Karnad, A \& Freeman, J. W. (2018). The biology and role of CD44 in cancer progression : therapeutic implication. Journal of Hematology, 11(64), 1-23. doi: 10.1186/s13045-018-0605-5.

Chen, M. L., Pittet, M. J., Gorelik, L., Flavell, R. A., Weissleder, R., von Boehmer, H., \& Khazaie, K. (2005). Regulatory T cells suppress tumor-specific CD8 T cell cytotoxicity through TGF-beta signals in vivo. Proceedings of the National Academy of Sciences of the United States of America, 102(2), 419-424. https://doi.org/10.1073/pnas.0408197102 
Cho, J., Chang, Y. H., Heo, Y. J., Kim, S., Kim, N. K., Park, J. O., Kang, W. K., Lee, J., \& Kim, K. M. (2018). Four distinct immune microenvironment subtypes in gastric adenocarcinoma with special reference to microsatellite instability. ESMO open, 3(3), e000326. https://doi.org/10.1136/esmoopen-2018-000326

Chu, P. G. dan Weiss, L. M. (2009) 'Modern immunohistochemistry', Cambridge Illustrated Surgical Pathology, pp. 489-490.

Daster, S., Eppenberger-Castori, S., Hirt, C., Zlobec, I., Delko, T., Nebiker, C. A., Soysal, S. D., Amicarella, F., lezzi, G., Sconocchia, G., Heberer, M., Lugli, A., Spagnoli, G. C., Kettelhack, C., Terracciano, L., Oertli, D., von Holzen, U., Tornillo, L., \& Droeser, R. A. (2014). High frequency of CD8 positive lymphocyte infiltration correlates with lack of lymph node involvement in early rectal cancer. Disease markers, 2014, 792183. https://doi.org/10.1155/2014/792183

de Vries, N. L., Swets, M., Vahrmeijer, A. L., Hokland, M., \& Kuppen, P. J. (2016). The immunogenicity of colorectal cancer in relation to tumor development and treatment. International Journal of Molecular Sciences, 17(7), 1030. https://doi.org/10.3390/ijms17071030

Du, L., Wang, H., He, L., Zhang, J., Ni, B., Wang, X., Jin, H., Cahuzac, N., Mehrpour, M., Lu, Y., \& Chen, Q. (2008). CD44 is of functional importance for colorectal cancer stem cells. Clinical cancer research : an official journal of the American Association for Cancer Research, 14(21), 6751-6760. doi:10.1158/10780432.CCR-08-1034.

Dushyanthen, S., Beavis, P. A., Savas, P., Teo, Z. L., Zhou, C., Mansour, M., Darcy, P. K., \& Loi, S. (2015). Relevance of tumor-infiltrating lymphocytes in breast cancer. BMC Medicine, 13, 202. https://doi.org/10.1186/s12916-015-0431-3

Hamilton, S. R., Bosman, F. T., Boffeta, P., Ilyas, M., Morreau, H., Nakamur, S. I., Quirke, P., Riboli, E., Sobin, L. H. (2010) WHO classification of tumours of the digestive system(4th ed). Lyon: IARC Press, pp. 131-146.

Holah, N. S., Aaid, H. A., Assad, N. Y., Elkhouly, E. A., \& Lasheen, A. G., (2017) Evaluation of the role of CD44 as a cancer stem cell marker in colorectal carcinoma: immunohistochemical study.Menoufia Medical Journal, 30, 174-183. doi: 10.4130/mmj. Mmj_151_16.

Idos, G. E., Kwok, J., Bonthala, N., Kysh, L., Gruber, S. B., \& Qu, C. (2020). The prognostic implications of tumor infiltrating lymphocytes in colorectal cancer: a systematic review and meta-analysis. Scientific Reports, 10(1), 3360. https://doi.org/10.1038/s41598-020-60255-4

Iseki, Y. Shibutani, M. Maeda, K. Nagahara, H. Fukuoka, T. Matsutani, S. Kashigawa, S. Tanaka, H. Hirakawa, K., \& Ohira, M. (2018). Infiltrating Lymphocytes (TILs) in colorectal cancer using hematoxylin and eosis (HE)-stained tumor sections', PLOS ONE, 13(4), 1-12. doi: 10.13/1/journal.pone.0192744.

Jackute, J., Zemaitis, M., Pranys, D., Sitkauskiene, B., Miliauskas, S., Bajoriunas, V., Lavinskiene, S., \& Sakalauskas, R. (2015). The prognostic influence of tumor infiltrating Foxp3(+)CD4(+), CD4(+) and CD8(+) T cells in resected non-small cell lung cancer. Journal of Inflammation, 12, 63. https://doi.org/10.1186/s12950015-0108-X

Khedr, R.A.E, Ghannam A.A.E, El-Rahidy. M. A.,\&El-Deen, A.A.S. (2016) The prognostic role of tumor infiltrating lymphocytes CD8 and FOXP3 and their impact on recurrence in breast cancer patients. Journal of Cancer Science \& Therapy, 8(7), 206-212. doi:10.4172/1948-5956.1000414. 
Ma, L., Dong, L., \& Chang, P. (2019). CD44v6 engages in colorectal cancer progression. Cell Death \& Disease, 10(1), 30. https://doi.org/10.1038/s41419018-1265-7

Ma, L., Jiang, T. (2013). Clinical implications of Ezrin and CD44 co-expression on breast cancer. Oncology Reports, 30, 1899-1905

Magee, J. A., Piskounova, E., \& Morrison, S. J. (2012). Cancer stem cells: impact, heterogeneity, and uncertainty. Cancer Cell, 21(3), 283-296. https://doi.org/10.1016/j.ccr.2012.03.003

Peng, G. L., Li, L., Guo, Y. W., Yu, P., Yin, X. J., Wang, S., \& Liu, C. P. (2019). $\mathrm{CD}^{+}$cytotoxic and FoxP3 ${ }^{+}$regulatory $\mathrm{T}$ lymphocytes serve as prognostic factors in breast cancer. American Journal of Translational Research, 11(8), 50395053.

Rawla, P. Sunkara, T. Barsouk, A. (2019) Epidemiology of colorectal cancer: incidence, mortality, survival, and risk factors, Gastroenterology Review, 14(2), 89-90. doi:105114/pg.2018.81072.

Wang, M., Zhao, J., Zhang, L., Wei, F., Lian, Y., Wu, Y., Gong, Z., Zhang, S., Zhou, J., Cao, K., Li, X., Xiong, W., Li, G., Zeng, Z., \& Guo, C. (2017). Role of tumor microenvironment in tumorigenesis. Journal of Cancer, 8(5), 761-773. https://doi.org/10.7150/jca.17648

Wang, Z., Tang, Y., Xie, L., Huang, A., Xue, C., Gu, Z., Wang, K., \& Zong, S. (2019). The prognostic and clinical value of CD44 in colorectal cancer: a metaanalysis. Frontiers in Oncology, 9, 309. https://doi.org/10.3389/fonc.2019.00309

West, N. R., McCuaig, S., Franchini, F., \& Powrie, F. (2015). Emerging cytokine networks in colorectal cancer. Nature reviews. Immunology, 15(10), 615-629. https://doi.org/10.1038/nri3896

Xu, C., Zhao, H., Chen, H., \& Yao, Q. (2015). CXCR4 in breast cancer: oncogenic role and therapeutic targeting. Drug Design, Development and Therapy, 9, 49534964. https://doi.org/10.2147/DDDT.S84932

Yang, L., \& Zhang, Y. (2017). Tumor-associated macrophages: from basic research to clinical application. Journal of Hematology \&Oncology, 10(1), 58. https://doi.org/10.1186/s13045-017-0430-2

Zhao, L. H., Lin, Q. L., Wei, J., Huai, Y. L., Wang, K. J., \& Yan, H. Y. (2015). CD44v6 expression in patients with stage II or stage III sporadic colorectal cancer is superior to CD44 expression for predicting progression. International Journal of Clinical and Experimental Pathology, 8(1), 692-701.

Zhou, Y., Xia, L., Wang, H., Oyang, L., Su, M., Liu, Q., Lin, J., Tan, S., Tian, Y., Liao, Q., \& Cao, D. (2017). Cancer stem cells in progression of colorectal cancer. Oncotarget, 9(70), https://doi.org/10.18632/oncotarget.23607

Ziai, J., Gilbert, H. N., Foreman, O., Eastham-Anderson, J., Chu, F., Huseni, M., \& Kim, J. M. (2018). CD8 ${ }^{+}$T cell infiltration in breast and colon cancer: a histologic and statistical analysis. PloS One, 13(1), e0190158. https://doi.org/10.1371/journal.pone.0190158 